\title{
Validation of SyS Data to Inform Surveillance of Health Disparities in Nebraska
}

\author{
Sandra Gonzalez ${ }^{\star 1,2}$, Ashley Newmyer ${ }^{1}$ and Ming $\mathbf{Q u}{ }^{1}$ \\ 'Division of Public Health, Epidemiology \& Informatics Unit, Nebraska Department of Health and Human Services, Lincoln, NE, USA; \\ ${ }^{2}$ University of Nebraska-Lincoln, Lincoln, NE, USA
}

\section{Objective}

This pilot study evaluates Nebraska Department of Health and Human Services (NDHHS) emergency department (ED) syndromic surveillance (SyS) data quality by cross-validating reported external cause of injury codes (ECODES) associated to racial/ethnic injury health disparities in Nebraska. The percent completeness of core data elements in SyS data and hospital discharge data (HDD) was also determined.

\section{Introduction}

Achieving health equality is a national priority. The surveillance of health disparities in minority populations is key for the advancement of health equality. However, the need for improvement in documentation of race and ethnicity has been identified across various public health data sets. Currently, due to the lack of reporting of race and ethnicity in HDD, the NDHHS mainly depends on analyses of the statewide Behavioral Risk Factor Surveillance System and Vital Records data for the surveillance of health disparities among minority populations. An alternative data set that might help inform the surveillance of health disparities is SyS data. This near-real-time electronic health record data is characterized by required core data elements that provide information about the date and time of patient encounter, treating facility, clinical information, and patient demographics.

Previously, we demonstrated statistically significant correlations between the 2011 and 2012 NDHHS ED SyS and ED HDD data for ICD9-CM ECODES corresponding to motor vehicle crash related injury, which is a relevant cause of health disparities. Our new objective was to determine the reporting consistency of ICD9-CM ECODES associated with other injury related health disparities between 2013 NDHHS SyS and HDD ED data. We also sought to determine if near-real-time ED and IP SyS data provide a more complete documentation of race and ethnicity than HDD.

\section{Methods}

Completeness of core data elements was assessed for 2013 NDHHS ED HDD and IP, and for 2013-2015 NDHHS SyS ED and IP data from Hospital A. Core data elements for the SyS and HDD datasets included facility ID, patient ID, visit ID, visit date, patient's age, patient's sex, patient's race, patient's ethnicity, patient's zip code of residence, and diagnostic codes. The completeness of chief complaint was also analyzed for SyS data. The timeliness of SyS data was determined by calculating the mean time in hours between patient visit and receipt of ED SS record at NDHHS. The consistency of ICD9-CM reporting was assessed by comparing 2013 NDHHS SyS ED to 2013 HDD ED data from Hospital A. The cross-validation focussed on 2 causes of racial/ethnic injury related health disparities: assault-related injury, and suicide and self-inflicted injury. The corresponding ICD9-CM ECODES were: E960-E969, E979 and E999.1 (assault-related injury), and E950-E959 (suicide and self-inflicted injury). Pearson correlation coefficients were used to compare the frequency distribution of monthly counts for the specified diagnostic codes.

\section{Results}

For Hospital A 2013 NDHHS SyS data, the percent of completeness of most core data elements was $100 \%$. The percent of completeness for race and ethnicity was $100 \%$ and $92 \%$ respectively for the 2013 SyS IP data and $100 \%$ and $0 \%$ for ED data. An improvement in the percent of completeness for ethnicity was observed for the 2015 IP and ED SyS data, with $100 \%$ for both the 2015 NDHHS ED and IP SyS data. On the other hand, for the 2013 ED and IP HDD data, while the completeness of most core data elements was $100 \%$, a $0 \%$ of completeness was observed for race and ethnicity. Statistically significant correlations were observed between Hospital A 2013 ED HDD and NDHHS ED SyS data for assault-related injury $(r=0.72$, $\mathrm{p}=0.008)$, and suicide and self-inflicted injury $(\mathrm{r}=0.76, \mathrm{p}=0.004)$. The timeliness of reporting was 12 hours for the 2013 NDHHS SyS ED data and 2 weeks for the SyS IP data.

\section{Conclusions}

Results suggest that NDHHS SyS 2015 data provides more complete documentation of race and ethnicity than HDD. In addition, significant correlations were observed for the conditions analyzed. Therefore, the ability to identify and describe injury inequalities can potentially be improved by using SyS ED data to complement the surveillance of health disparities. These results also indicate that SyS ED data could also be used for the timely identification and monitoring of intentional injuries in Nebraska.

\section{Keywords}

syndromic surveillance; emergency department; health disparities; injury

\section{Acknowledgments}

Other contributors: Bryan Buss, DVM, MPH; Gary White, and Anthony Zhang, MA

\section{*Sandra Gonzalez}

E-mail: Sandra.Gonzalez@nebraska.gov 